\title{
SIMPLIFIED CLOSED-FORM SOLUTION FOR THE DETERMINATION OF THE MOMENT-CURVATURE RESPONSE OF A CIRCULAR RC SECTION
}

\author{
Domenico Raffaele ${ }^{1}$, Roberto Gentile ${ }^{1}$, and Mauro Mezzina ${ }^{1}$ \\ ${ }^{1}$ DICATECh, Politecnico di Bari, Bari, Italy \\ e-mail: \{domenico.raffaele, roberto.gentile, mauro.mezzina\}@poliba.it
}

Keywords: simply supported bridges; reinforced concrete; seismic vulnerability; momentcurvature.

\begin{abstract}
A seismic vulnerability analysis of a multi-span simply supported bridge is often based on the seismic response of the most critical pier. This response is influenced by different collapse modes (flexural, shear, second order effects, lap-splice of longitudinal bars or their buckling). Among these the flexural behaviour is important and it's known if the equivalent plastic hinge length and the Moment-Curvature law of the fixed end are given. This paper provides a closed-form dimensionless solution to obtain a 5 point Moment-Curvature diagram for circular RC section. The solution is based only on three parameters: dimensionless axial force, mechanical percentage of longitudinal reinforcement, geometrical percentage of transversal reinforcement. A numerical example is presented to test the solution comparing it with a FEM analysis.
\end{abstract}




\section{Introduction}

Seismic vulnerability is an actual theme in engineering and in particular great attention has been devoted to bridge structures in order to perform vulnerability inventory at a regional scale. In fact for example the major part of the Italian RC bridges was built around the 1960s referring to codes with no "anti seismic philosophy" [1] and studies based on the monitoring of bridges [2] support the need of the above mentioned inventory. Moreover a bridge can often be a crucial node of a transport web and thus it's performance has to be guaranteed even in the moments next to an earthquake [3]. Hence having an inventory of the structural performances of the bridges in a region is a central question in order to plan mitigation actions. Considering the large amount of the structures to analyze and the shortage of the resources to do that, it is impossible to perform detailed analysis to obtain capacity curves (e.g. Pushover Analysis). Usually regional scale analysis resorts to typological or semi empirical vulnerability methods but in this paper it's used a procedure that is based on rational mechanics with whom the ultimate displacement of a multi span bridge can be obtained with small computational effort and with few input data. Basing on the so called "Simplified Pushover Analysis" [4], instead of performing a full structural analysis under pushing loads, the vulnerability of the bridge is directly expressed by the capacity curve of an equivalent SDoF system. Moreover, given that the energy dissipation capacity of a simply supported bridge is concentrated in the piers and the deck usually remains elastic, in the simplified analysis only the piers contribute to model the SDoF system. The most critical pier of the bridge can be defined as the one that exibits the greater seismic demand/capacity ratio at a given limit state (fig.1). Often the capacity curve of the most critical pier is a good approximation of the capacity curve of the whole bridge. In this procedure [5] the capacity curve of the most critical pier, expressed in terms of Moment-Displacement $(M-\delta)$, is obtained taking into account the flexural behaviour (Moment-Curvature curve of the base section) and all the other collapse modes (shear, second order effects, lap-splice of longitudinal bars or their buckling) that can anticipate the flexural collapse. In this paper is proposed a procedure with which the Moment-Curvature $(M-\varphi)$ relationship of an RC section is calculated by defining the position of 5 characteristic points. Each of these is defined in a closed form depending on 3 parameters: dimensionless axial force, mechanical percentage of longitudinal reinforcement, geometrical percentage of transversal reinforcement. The basic concept is that knowing only a few parameters like geometry and amount of reinforcement, for example through a simple inspection, it is possible to perform a first level vulnerability analysis of a group of circular column multi span simply supported bridges. The approach herein proposed is based on realistic stress-strain relationships for both concrete and steel and its reliability is proved analyzing a numerical example and comparing it with a FEM non linear analysis.

\section{Summary of the procedure}

The Moment-Curvature relationship is obtained defining the position of 5 characteristic points (defined later). Moment and curvature of each of these points are predicted through a 5 th grade polynomial. It was created a database of Moment-Curvature analysis conducted with the software $K S U R C$ [6]. The results are processed in MATLAB in order to define the characteristic points of each $(M-\varphi)$ curve. Finally the polynomials are obtained with a least squares method linear regression. 


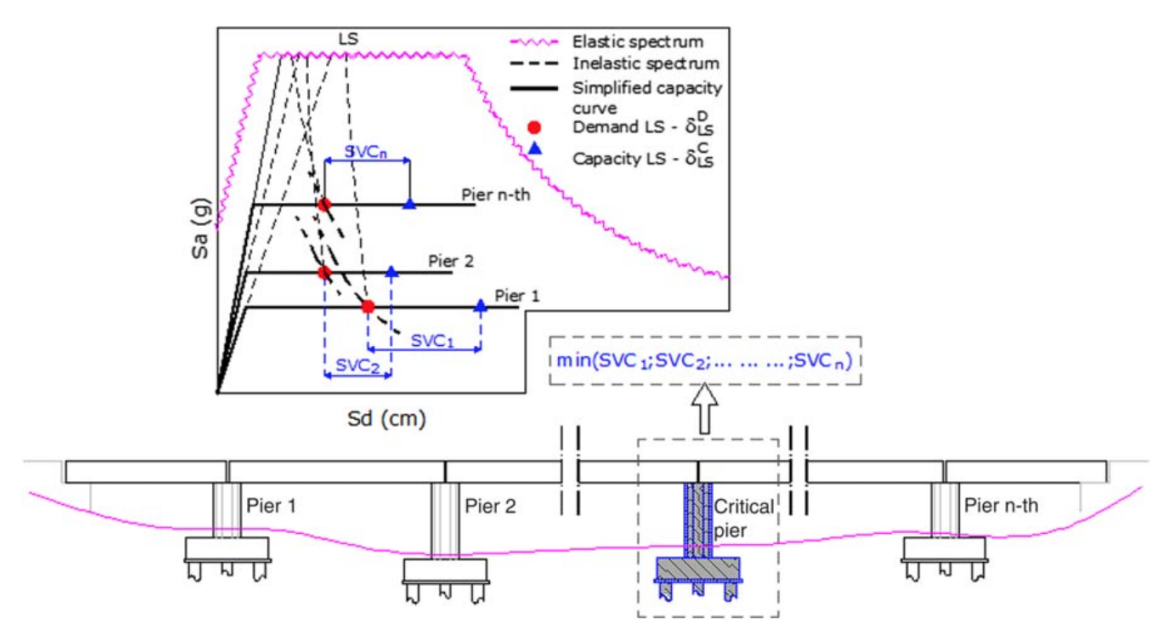

Figure 1: Determination of the critical pier.

\section{Selecting the problem parameters}

The parameters involved in the definition of the flexural behaviour of an RC circular section are several: radius $R$, clear cover $c$, normal force $N$, compression strength of concrete in unconfined conditions $f_{c}$, tension strength of concrete $f_{c t}$, concrete's modulus of elasticity $E_{c}$, yielding strength of longitudinal and transversal reinforcement $f_{y s}, f_{y h}$, moduli of elasticity of the steel $E_{s}, E_{h}$, number and diameter of longitudinal reinforcement $n_{l}, d_{l}$, diameter and spacing of transversal reinforcement $d_{h}, s$. It is clear that, for the scope of this work, it's inconvenient to have a so large number of parameters and for this reason some hypothesis have been made:

- concrete tension strength $f_{c t}$ has been related to its compression strength according to a relationship contained in the Italian code [7]:

$$
f_{t}=0.3 f_{c}^{\frac{2}{3}}
$$

Finally the relation

$$
f_{c t}=1.2 f_{t}(N T C 08)
$$

is used in order to consider the flexural tension strength;

- the transversal reinforcement steel has the same characteristics of the one used for longitudinal reinforcement. So

$$
f_{y h}=f_{y} \quad \text { and } \quad E_{s h}=E_{s}
$$


- longitudinal reinforcement is composed by $n_{l}$ evenly distributed bars of equal diameter $d_{l}$. So the total area of longitudinal steel is

$$
A_{s}=\frac{n_{l}\left(\pi d_{l}^{2}\right)}{4}
$$

- the clear cover is proportional to the radius of the section according to:

$$
c=0.05 R \text {. }
$$

The remaining parameters of the problem are grouped into 3 dimensionless parameters (dimensionless axial force $\nu$, mechanical percentage of longitudinal reinforcement $\omega$, geometrical percentage of transversal reinforcement $\rho$ ) so that a circular RC section is completely defined by them:

$$
\begin{gathered}
\nu=\frac{N}{\pi f_{c} R^{2}} \\
\omega=\frac{A_{s} f_{y}}{\pi f_{c} R^{2}} \\
\rho_{s p}=\frac{4 A_{s p}}{(2 R-2 c) s} .
\end{gathered}
$$

The idea is that a variation in $\nu$ can be seen as a variation in the normal force $N$, the concrete compression strength $f_{c}$ or the radius $R$ and similarly for the equations 7 and 8 .

\section{Parameters' ranges}

In order to create the database cited in par.2 it's been selected a plausible range for each of the three parameters $(\nu, \omega, \rho)$. Then, picked up enough discrete values in these intervals, it's been conducted a Moment-Curvature analysis for each combination (462 analysis) of them using the software $K S U \_C$.

\subsection{Axial force}

It's been considerated the entire range of variation for this parameter (from 0 to 1 ) and the 11 chosen discrete values are:

$$
\nu=\{0,0.1,0.2,0.3,0.4,0.5,0.6,0.7,0.8,0.9,1\} .
$$

For the analysis in $K S U \_R C$ it's been fixed $R=1 \mathrm{~m}$ and $f_{c}=31.83 \mathrm{Mpa}$ so the value of $N$ corresponding to $\nu$ it's been calculated with eq. 6 


\subsection{Longitudinal reinforcement}

It's been chosen a range between 0.05 and 0.8 and in particular the 6 values:

$$
\omega=\{0.05,0.1,0.2,0.4,0.6,0.8\}
$$

For the numerical analysis it's been fixed $f_{y}=450 \mathrm{MPa}$ and $n_{l}=22$ so the cross sectional area of a single rebar it's been calculated using eqs. 7 and 4 .

\subsection{Transversal reinforcement}

The range of this parameter goes from 0 to 0.01 and in particular the 7 values:

$$
\rho_{s p}=\{0,0.001,0.002,0.004,0.006,0.008,0.010\} .
$$

Fixing the spacing of the stirrups $(s=0.1 \mathrm{~m})$, according to the fixed values mentioned in pars 4.1 and 4.2 and knowing the value of the clear cover (eq.5) the cross sectional area of the stirrup has been calculated using eq, 8 .

\section{Execution of the analysis}

For each combination of the parameters mentioned in par4 (hence $11 \times 6 \times 7=462$ ) it's been conducted a Moment-Curvature analysis using the software $K S U \_R C$ whose reliability has been proved [6]. The cross section is divided into fibers and a stress-strain relationship is assigned to each of them. Concrete follows the relationship proposed by Mander [8] in order to model the different behaviour (fig.2) of concrete in the core (confined) and in the cover (unconfined). In order to model the behaviour of steel $K S U \_C$ uses a stress-strain relationship, proposed by the owner of the software, that brings to results in good agreement with the experiments. The curve (fig. 3 is assumed linear up to the yielding, has a constant tension until a strain of $K_{1}$ times the yielding strain and then is parabolic. The peak of the parabola corresponds to a strain of $K_{2}$ times the yielding strain and the ultimate point corresponds to a strain of $K_{3}$ times the yielding strain. The peak strength is equal to $K_{4}$ times the yielding strength. For this study the values used are such that the curve best approximates the real behaviour of the most common italian steel. In particular: $K_{1}=10, K_{2}=30, K_{3}=55, K_{4}=1.3$.

The output of the software is a 7-column array containing curvature, moment, neutral axis, strain in the furthermost fiber of the concrete core, strain of the extreme tension rebar and axial force.

\section{Post processing}

It's been projected a MATLAB function that analyzes each $(M-\varphi)$ curve in order to obtain a 5-point curve (an example can be seen in fig 5). The function input is the 7-column array obtained from the output of $K S U \_R C$. The characteristic points of the $(M-\varphi)$ curve chosen are:

Cracking If the curve presents a softening branch at cracking this point is exactly the start of this branch. If else this branch isn't appreciable, and this can occur for great values of the axial force, the cracking point assumed is the one that induces a strain of $\varepsilon_{c t}$ in the furthermost concrete fibre in the section (fig.47. In particular the function finds the value of $\varepsilon_{c}$ that satisfy eq: 


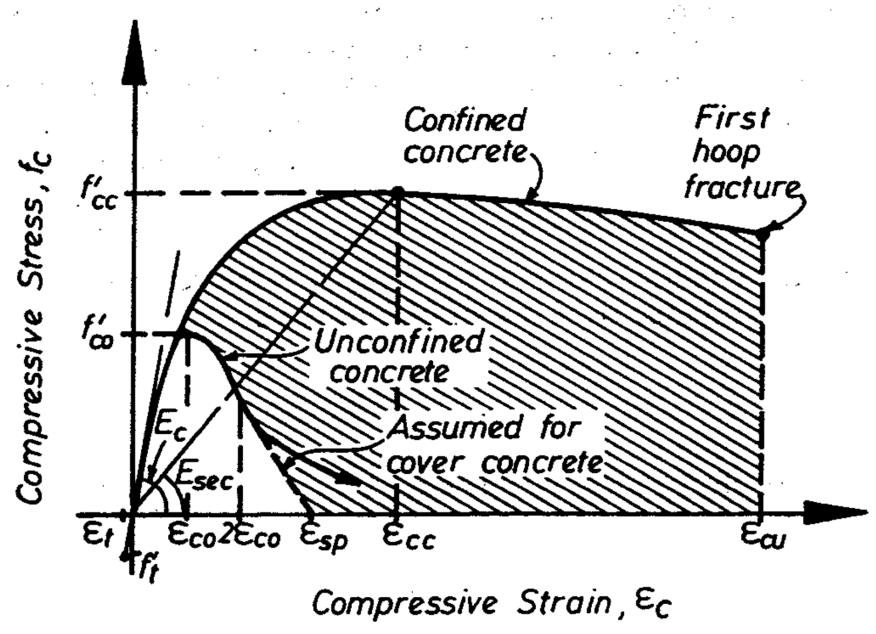

Figure 2: Mander stress-strain relationship for confined and unconfined concrete.

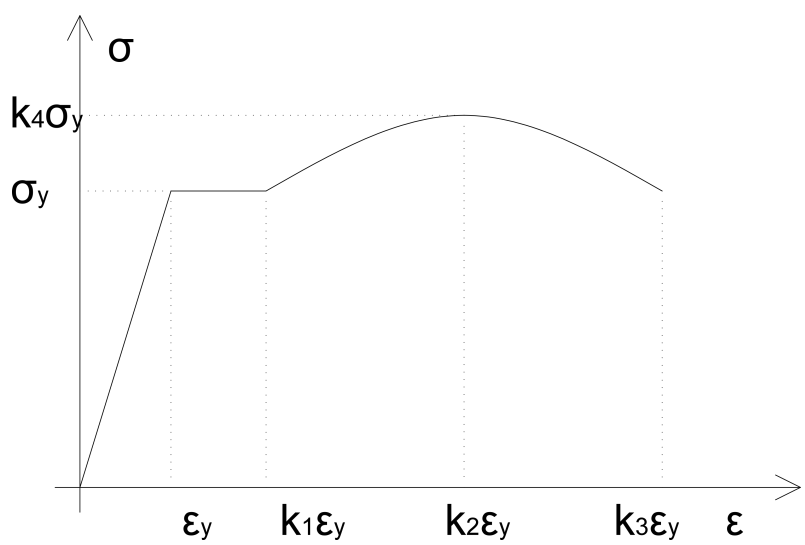

Figure 3: Stress-strain relationship for steel.

$$
\frac{D-x}{x-c} \varepsilon_{c}=\varepsilon_{c t}
$$

where

$$
\varepsilon_{c t}=\frac{f_{c t}}{E_{c}}
$$

First Yield This point is defined as the point on $(M-\varphi)$ curve when the reinforcement furthest from the neutral axis attains the first yield, or when extreme concrete compression fibre attains a strain of 0.002 , which ever occurs first [9].

Spalling The point of the $(M-\varphi)$ response that induces a strain of 0.0045 in the extreme fiber of the cover concrete (fig. 4) according to eq: 


$$
\frac{x}{x-c} \varepsilon_{c}=\varepsilon_{s p}
$$
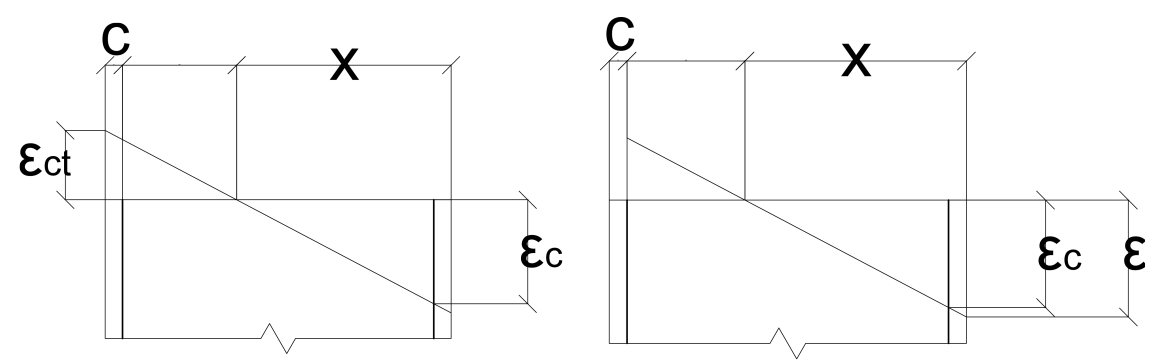

Figure 4: Strain state of the section at cracking and spalling.

Peak of confined concrete Defined as the condition that causes, in the extreme core concrete fibre, a strain corresponding to its peak in the Mander constitutive relation.

Collapse Simply the last point of the $(M-\varphi)$ curve. It can represent both the failure due to reaching the ultimate strain in confined concrete or in the extreme tension rebar.

Finally the curves are tranformed in dimensionless form according to:

$$
\begin{gathered}
m=\frac{M}{\pi R^{3} f_{c}} \\
\chi=1000 \varphi R .
\end{gathered}
$$

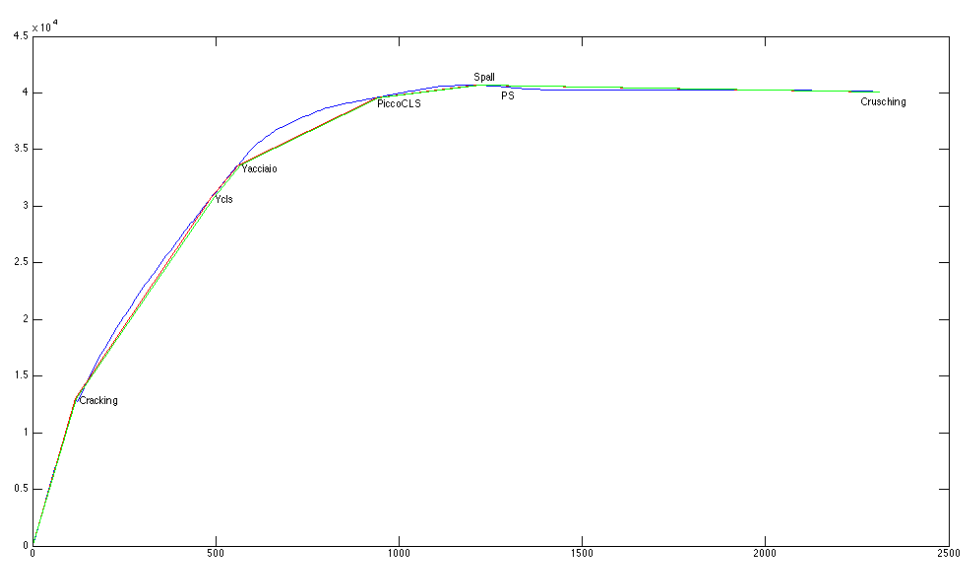

Figure 5: 5-point Moment-Curvature relationship. 


\section{Curve fitting}

Knowing the values of the characteristic moments and curvatures for each tern of parameters $\nu, \omega, \rho$ it's been possible to find a 5th-grade polynomial in these three variables that best fit the data basing on a least square method linear regression. Notice that the terns with $\rho=0$ are studied only for control purposes and so they are excluded from the fitting. The structure of the polynomials (eq 17) is shown in appendix A. It is obvious that the manual use of these functions is practically impossible but it will be easy to use them with a simple software.

\section{Numerical application}

With the aim of demonstrating that the simplified procedure to evaluate the Moment-Curvature of an RC section herein proposed can be used as a reliable input for the assessment of a simply supported bridge (e.g. assessing the most critical pier) a numerical validation of it is presented. The response of the base section of the most critical pier is predicted according to the characteristic polynomials of appendix A and compared to a numerical analysis conducted with $K S U \_C$ (fig 6). Basing on a linearisation of the $(M-\varphi)$ relationship, the Moment-displacement curve of the pier has been calculated according to a simplified procedure proposed by Raffaele et al. [5] named VulPil. According to this procedure knowing the $(M-\varphi)$ curve of the base section of the pier and the length of the plastic hinge it can be calculated the flexural $(M-\delta)$ relationship. Then this curve can be "corrected" in order to take into account the different collapse modes. It is important to notice that in this work only the $(M-\varphi)$ curve is to be validated and so the benchmark case is calibrated in such a way that it is only governed by the flexural behaviour. The $(M-\delta)$ curve is compared to the results of a FEM analysis performed using SAP2000 V17. In SAP the pier has been modeled by means of 12 "beam" elements each one discretized into 52 fibers. It has been conducted a pushover analysis under a single horizontal load on the top of the pier. The constitutive laws used in SAP can be seen in figs.2 and 3 . According to the simplified procedure used the behaviour of the pier depends on the presence of seismic restraints and this can be taken into account by considering the effective mass pertaining to the pier. For this reason it is necessary to consider two different models, one for the transversal and one for the longitudinal direction (they differ only for the axial force acting on the pier). In table 1 the geometric and mechanical parameters for the pier chosen for this example. It should be noted that the example chosen is identical to the one chosen by [5]. The reason of doing that is to show the improvement of the $(M-\delta)$ relationship prediction due to the more refined $(M-\varphi)$ curve. In fact the simplified $(M-\delta)$ analysis in this example is different from the VulPil analysis only in the definition of the $(M-\varphi)$ curve. The resulting capacity curve for the transversal direction is represented in fig.7/in which the curve calculated with SAP2000 has been transformed in a bilinear curve in order to simplify the comparison.

Looking at the comparison it can be seen that the simplified procedure is close to the refined numerical solution. There is an important improvement in the prediction of the yielding displacement and the maximum moment, with respect to the VulPil procedure, due to the better definition of the $(M-\varphi)$ curve. Conversely it can be seen an over prediction of the ultimate displacement of the pier with respect to the SAP2000 analysis. In table 2 is presented a numerical comparison of the results in terms of ultimate moment, ultimate displacement and displacement ductility. 


\begin{tabular}{cccccccc}
\hline & $\begin{array}{c}L \\
{[\mathrm{~m}]}\end{array}$ & $\begin{array}{c}D \\
{[\mathrm{~m}]}\end{array}$ & $\begin{array}{c}c \\
{[\mathrm{~m}]}\end{array}$ & $\begin{array}{c}n_{l} \\
{[-]}\end{array}$ & $\begin{array}{c}d_{l} \\
{[\mathrm{~mm}]}\end{array}$ & $\begin{array}{c}d_{h} \\
{[\mathrm{~mm}]}\end{array}$ & $\begin{array}{c}s \\
{[\mathrm{~mm}]}\end{array}$ \\
\hline Transversal & 6 & 2 & 0.05 & 30 & 26 & 10 & 100 \\
Longitudinal & 6 & 2 & 0.05 & 30 & 26 & 10 & 100 \\
\hline \hline & $f_{c}$ & $f_{c t}$ & $f_{y s}$ & $N$ & $\nu$ & $\omega$ & $\rho_{s p}$ \\
& {$[M P a]$} & {$[M P a]$} & {$[M P a]$} & {$[K N]$} & {$[-]$} & {$[-]$} & {$[-]$} \\
\hline Transversal & 20 & 3 & 450 & 13870 & 0.678 & 0.107 & 0.009 \\
Longitudinal & 20 & 3 & 450 & 17847 & 0.678 & 0.107 & 0.009 \\
\hline
\end{tabular}

Table 1: Geometric and mechanical properties of the pier.

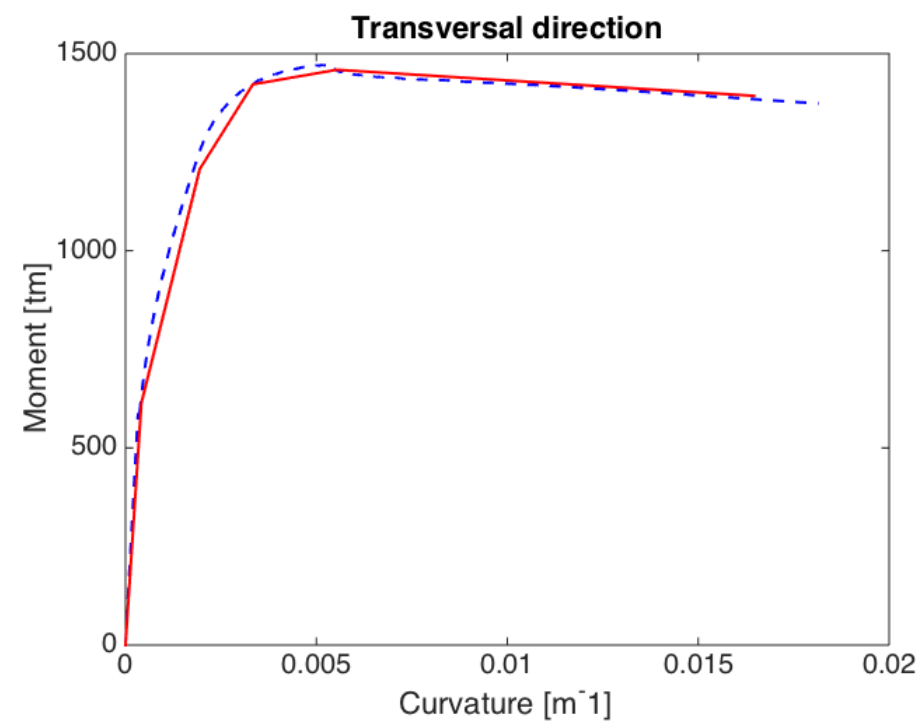

Figure 6: Moment-Curvature: comparison between KSU_RC and the simplified procedure, transversal direction.

\begin{tabular}{cccc}
\hline & $\Delta M_{u}$ & $\Delta \delta_{u}$ & $\Delta \mu$ \\
& {$[\%]$} & {$[\%]$} & {$[\%]$} \\
\hline Transversal & 3,23 & 27,36 & 13,14 \\
Longitudinal & 2,63 & 17,43 & 6,82 \\
\hline
\end{tabular}

Table 2: Simplified analysis and refined FEM analysis. Numerical comparison of the results. 


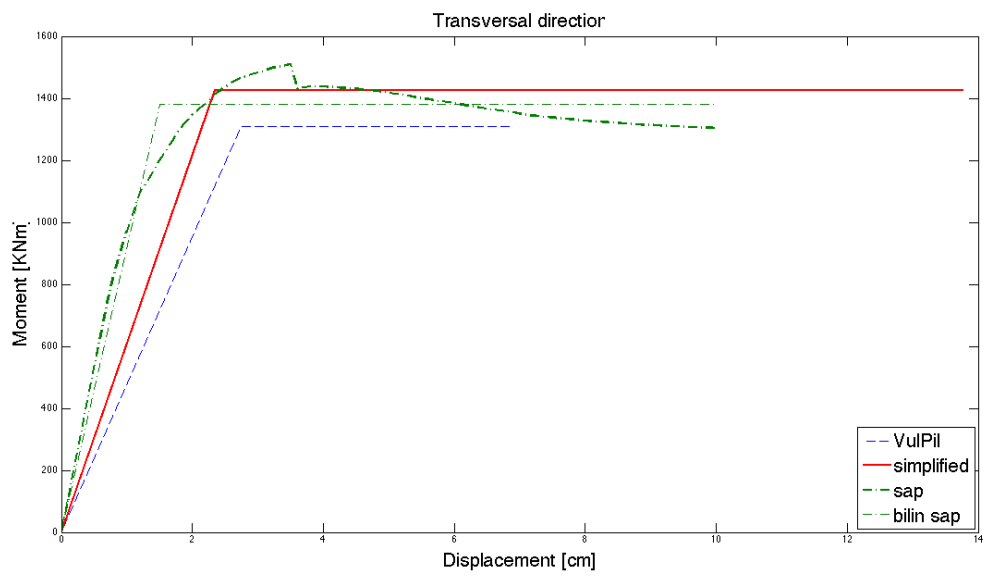

Figure 7: Comparison between capacity curves, transversal direction.

\section{Conclusions}

The need to develop procedure for performing the vulnerability inventory of the bridges in a specific region is a central question in order to plan mitigation actions. Most of the simplified procedures aimed to the assessment of a simply supported bridge are based on the knowledge of the flexural behaviour of the base section of the most critical pier. In this paper is proposed a polynomial closed form solution to obtain a 5-point Moment Curvature curve of an RC section knowing only few parameters. After describing the whole procedure used to obtain the above mentioned polynomials the procedure has been applied to a benchmark case and the solution has been compared to a FEM analysis. The differences in the results found with the simplified procedure and the FEM analysis are quite small, especially because they are obtained with really few input data.

\section{Acknowledgements}

The research presented in this article was partially funded by the Department of Civil Protection, Project ReLUIS-DPC 2014-2018

\section{REFERENCES}

[1] D. Raffaele, F. Porco, G. Uva, A. Fiore, Simplified assessment of seismic retrofitting interventions on RC circular piers in multi-span simply supported bridges. Proceedings of the 7th International Conference on Bridge Maintenance, Safety and Management, Shanghai, China; 7-11/07/2014, Taylor and Francis ISBN 978-113800103-9.

[2] G. Uva, G. Porco, F. Porco, A. Fiore, Monitoring and safety for prestressed bridge girders by SOFO sensors. Journal of Civil Structural Health Monitoring (2013), vol. 3, N1, p. 3-18, ISSN: 2190-5452, DOI: 10.1007/s13349-012-0029-9.

[3] D. Raffaele, G. Uva, F. Porco, A. Fiore, About of seismic capacity of bridge piers: A simplified approach. Proceedings of the 7th International Conference on Bridge Maintenance, Safety and Management, Shanghai, China; 7-11/07/2014, Taylor and Francis ISBN 978-113800103-9. 
[4] B. Borzi, R. Pinho, H. Crowley, Simplified vulnerability analysis for large scale assessment of RC buildings. Engineering Structures, 2007.

[5] D. Raffaele, F. Porco, A. Fiore, G. Uva, Simplified vulnerability assessment of reinforced concrete circular piers in multi-span simply supported bridges, Structure and Infrastructure Engineering, 2014 vol. 10, N8, p. 950-962, ISSN: 1744-8980, DOI:10.1080/15732479.2013.772642.

[6] A. Esmaeily, R.J Peterman Performance analysis tool for reinforced concrete members. International Congress on Civil Engineering, 2005.

[7] DM 14/01/2008, Norme tecniche per le costruzioni. Gazzetta ufficiale n.29, Roma, Italy, 2008.

[8] J.B. Mander, M.J.N. Priestley, R. Park, Theoretical stress-strain model for confined concrete. Journal of Structural Engineering ASCE, 1988.

[9] M.J.N. Priestley, G.M. Calvi, M.J. Kowalsky, Displacement-based seismic design of structures. IUSS Press, Pavia, Italy, 2007.

\section{A Characteristic points polynomials}

$$
\begin{array}{r}
m_{\text {char }}\left(\text { or } \chi_{\text {char }}\right)=a_{1} \nu^{5}+a_{2} \nu^{4} \omega+a_{3} \nu^{4} \rho_{s p}+a_{4} \nu^{4}+a_{5} \nu^{3} \omega^{2}+a_{6} \nu^{3} \omega \rho_{s p}+a_{7} \nu^{3} \omega+ \\
+a_{8} \nu^{3} \rho_{s p}^{2}+a_{9} \nu^{3} \rho_{s p}+a_{10} \nu^{3}+a_{11} \nu^{2} \omega^{3}+a_{12} \nu^{2} \omega^{2} \rho_{s p}+a_{13} \nu^{2} \omega^{2}+a_{14} \nu^{2} \omega \rho_{s p}^{2}+ \\
+a_{15} \nu^{2} \omega \rho_{s p}+a_{16} \nu^{2} \omega+a_{17} \nu^{2} \rho_{s p}^{3}+a_{18} \nu^{2} \rho_{s p}^{2}+a_{19} \nu^{2} \rho_{s p}+a_{20} \nu^{2}+a_{21} \nu \omega^{4}+ \\
+a_{22} \nu \omega^{3} \rho_{s p}+a_{23} \nu \omega^{3}+a_{24} \nu \omega^{2} \rho_{s p}^{2}+a_{25} \nu \omega^{2} \rho_{s p}+a_{26} \nu \omega^{2}+a_{27} \nu \omega \rho_{s p}^{3}+a_{28} \nu \omega \rho_{s p}^{2}+ \\
+a_{29} \nu \omega \rho_{s p}+a_{30} \nu \omega+a_{31} \nu \rho_{s p}^{4}+a_{32} \nu \rho_{s p}^{3}+a_{33} \nu \rho_{s p}^{2}+a_{34} \nu \rho_{s p}+a_{35} \nu+ \\
+a_{36} \omega^{5}+a_{37} \omega^{4} \rho_{s p}+a_{38} \omega^{4}+a_{39} \omega^{3} \rho_{s p}^{2}+a_{40} \omega^{3} \rho_{s p}+a_{41} \omega^{3}+a_{42} \omega^{2} \rho_{s p}^{3}+ \\
+a_{43} \omega^{2} \rho_{s p}^{2}+a_{44} \omega^{2} \rho_{s p}+a_{45} \omega^{2}+a_{46} \omega \rho_{s p}^{4}+a_{47} \omega \rho_{s p}^{3}+a_{48} \omega \rho_{s p}^{2}+a_{49} \omega \rho_{s p}+ \\
+a_{50} \omega+a_{51} \rho_{s p}^{5}+a_{52} \rho_{s p}^{4}+a_{53} \rho_{s p}^{3}+a_{54} \rho_{s p}^{2}+a_{55} \rho_{s p}+a_{56} .
\end{array}
$$

